\title{
The whodunit of cancer
}

\author{
The Emperor of All Maladies: A Biography \\ of Cancer \\ Siddhartha Mukherjee \\ Scribner; 2010.
}

I $\mathrm{n}$ this epic volume, Dr. Siddhartha Mukherjee, a young medical oncologist with a strong academic pedigree at New York-Presbyterian Hospital, delivers what the title promises, a nontoxic and educational cocktail for the literate lay public and for those in the helping professions interested in cancer.

The volume is a tour de force navigating the course from early papyrus records in Egypt to modern epigenetics. His history of karkinos (the crab) spans ancient Egypt and Greece, tracking each new development in cancer through to molecular medicine and the promise of genomics for cancer treatment. The story is told with a very human voice through his personal encounters as a young oncologist/clinical scientist confronting the relentless march of his patients' suffering, death and yes, occasional triumphs. He throws in a good measure of the history of cancer medicine - albeit with a distinctly American tilt - all written in very well-crafted and articulate prose. The journey takes the reader down many interesting historical alleys, $\mathrm{cul}$ de sacs and personalities. A little weighty (well north of 500 pages), it nevertheless reads at times like a whodunit of cancer.

$\mathrm{He}$ also forays into the realm of treatments, moving from primitive early chemotherapy based on dyes, to the tragic emergence of radiation therapy (which kills many of its originators) and radical procedures versus minimal procedures. An intriguing paradox emerges in that successful breakthroughs in cancer have often occurred where bold physicians intentionally took reckless chances to achieve progress against this cunning and relentless enemy. These bold individuals have succeeded in making progress, usually limited progress. The relatively few true breakthroughs with durable cures are canvassed, including the treatment of childhood leukemia, Hodgkins lymphoma and testicular cancer (in younger men), and the recent emergence of genetic pathway analysis and molecular oncology with improved survival classes of patients with particular pathway signatures who respond to particular biological agents.

In treating cancer, we are all playing with fire. To make a systematic dent against this formidable disease, initial treatments must be refined by individuals who work to calibrate the new treatment with a standardized, minimum requirement to achieve the optimal outcome. For example after spending years with long radiation fractions as the radiation treatment of choice for women with breast cancer, Tim Whelan and colleagues have illustrated that short fractions may offer maximal tumour control while minimizing toxicity. ${ }^{1}$

In the past two decades significant and lasting improvements in overall survival and mortality reductions are apparent for some major and minor cancers. The development of new biologicals, like imatinib mesylate (Gleevec) and its analogs to treat chronic myelogenous leukemia and stromal tumours; the terrific story of Dennis Slaman, Genentech and the emergence of the Her-2 Neu receptor as an important pathway for "personalizing" breast cancer treatments with trastuzumab (Herceptin), are well documented along with a growing array of prognostic markers for cancer. Mukherjee sketches the rapid proliferation to hundreds of proto oncogenes, tumour suppressor genes and important pathways and switches in the triggering of mutation and the inhibition of particular cancer types.

The volume is careful to identify the political imperative of mobilizing for "a cure" while categorically dismissing the implausible, monolithic hammer of "a cure." Some of the simpler follies of earlier artificial environmental exposures like asbestos are now prevented through regulated exposure reduction and public health measures. The tobacco epidemic is one of these well-told stories.

He also recounts with style, how in

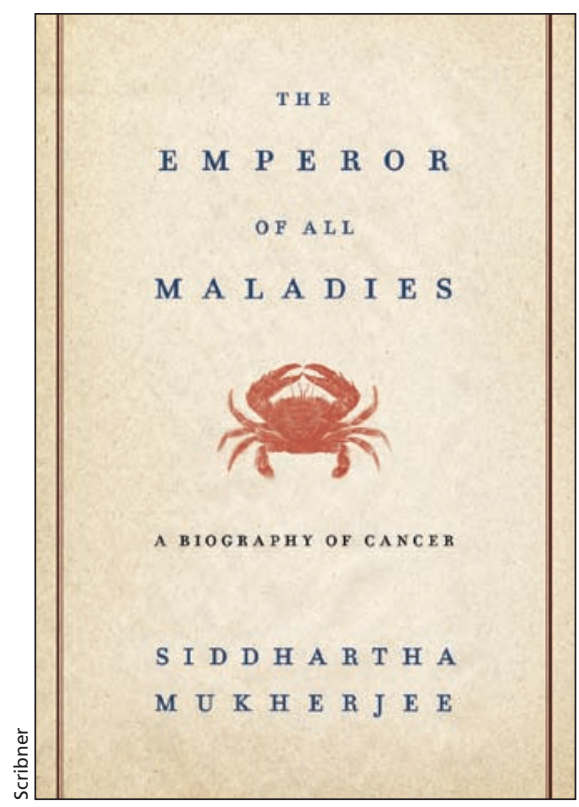

the last two decades, we have started making gains for several common and less common cancers. These improvements have been driven by a multitude of factors including the decline in tobacco consumption, some improved dietary practices, the emergence of screening programs for breast, colon, and cervical cancer, and of course the emergence of many better and more precisely targeted therapeutic interventions in surgery, radiation and chemotherapy have contributed as documented in some of the few modelling studies of these interventions on mortality and survival. Perhaps less well told is how these discoveries are spread or aren't spread systematically into population-wide cancer control programs through public policy informed by best evidence and better health care coverage for our populations.

One could easily see this volume resurfacing in an updated, shortened version, perhaps with a less Americentric view of the biography, and it could easily emerge as a text with a global reach and legacy.

\section{Terrence Sullivan PhD}

Chief Executive Officer

Cancer Care Ontario

Toronto, Ont.

\section{Reference}

1. Whelan TJ, Pignol JP, Levine MN, et al. Longterm results of hypofractionated radiation therapy for breast cancer. N Engl J Med 2010;362:513-20.

CMAJ 2011. DOI:10.1503/cmaj.110225 